\title{
Global stability analysis of a compressible turbulent flow around a high-lift configuration
}

\author{
M. C. Iorio * \\ Airbus Operations S.L., P John Lennon S/N, 28906 Getafe Madrid Spain. \\ L. M. González \\ Universidad Politécnica de Madrid, Avda. Arco del Triunfo 4, 28004 Madrid Spain. \\ A. Martínez-Cava \\ Airbus Group Innovations UK, Filton 20, Golf Course Lane BS347QW, Filton UK.
}

The purpose of this work is to analyze a complex high lift configuration for which significant regions of separated flow are present. Current state of the art methods have some difficulty to predict the origin and the progression of this separated flow when increasing the angle of attack. The mechanisms responsible for the maximum lift limit on multielement wing configurations are not clear; this stability analysis could help to understand the physics behind the phenomenon and to find a relation between the flow separation and the instability onset. The methodology presented herein consists in the computation of a steady baseflow solution based on a finite volume discretization and a proposal of the solution for a generalized eigenvalue problem corresponding to the perturbed and linearized problem. The eigenvalue problem has been solved with the Arnoldi iterative method, one of the Krylov subspace projection methods. The described methodology was applied to the NACA0012 test case in subsonic and in transonic conditions and, finally, for the first time to the authors knowledge, on an industrial multi-component geometry, such as the A310 airfoil, in order to identify low frequency instabilities related to the separation. One important conclusion is that for all the analyzed geometries, one unstable mode related to flow separation appears for an angle of attack greater than the one correspondent to the maximum lift coefficient condition. Finally, an adjoint study was carried out in order to

*Research Engineer, Airbus Operations S.L., maria-chiara.iorio@airbus.com. 
evaluate the receptivity and the structural sensitivity of the geometries, giving an indication of the domain region that could be modified resulting in the biggest change of the flowfield.

\section{Nomenclature}

$\overline{\{\}}$ Basic flow component

\{\} Perturbation component

$\widehat{\{\}}$ Amplitude function

$\alpha \quad$ Angle of attack

A Jacobian Matrix

B Volume matrix

c Chord

$C_{l} \quad$ Lift coefficient

$C_{v} \quad$ Specific heat capacity

$\varepsilon \quad$ Parameter

E Total specific energy

$f \quad$ Frequency

$\overline{\overline{\mathbf{F}}} \quad$ Flux density vector

$M \quad$ Mach number

n Number of subdomains faces

n Normal direction to the boundary

$N \quad$ Number of nodes of the domain

$N_{v} \quad$ Number of fluid dynamic variables

$\widetilde{\nu} \quad$ Turbulent kinematic viscosity

$q \quad$ Vector of conservative variables

$Q \quad$ Steady solution

$R \quad$ Radius of the domain

R Residual

Re Reynolds number

$\rho \quad$ Density

$S \quad$ Structural sensitivity

$\tau$ Pseudo-time

$t \quad$ Time

$T$ Temperature 
$\mathbf{U}^{F} \quad$ Fluxes over the domain boundaries

v Velocity vector, with components $(u, v, w)$

$x \quad$ Spatial coordinate, $x_{1}=x, x_{2}=y, x_{3}=z$

$\omega$ Complex eigenvalue, with components $\left(\omega_{r}, \omega_{i}\right)$

$\omega_{i} \quad$ Perturbation frequency

$\omega_{r} \quad$ Amplification/damping rate of linear perturbation

$\Omega \quad$ Fluid domain

Subscript

adj Adjoint

$d \quad$ Dimensional

$\max$ Maximum

sep Separation

$S L \quad$ Shear layer

Superscript

$H \quad$ Hermitian

\section{Introduction}

Nowadays, high-lift devices are used on airplanes due to the possibility of achieving shorter take-off and landing runs and reducing the angle of attack near the minimum flight speed. Smith ${ }^{1}$ summarizes the superior lifting capability of multi-element wings over single-element wings. The main advantage is the reduction of adverse pressure gradients due to higher velocities, and hence circulations, generated by upstream elements. In figure 1, the lift curve behavior is illustrated for increasing incidences, showing an increase of the lift on the main element and on the slat, while a decrease of the lift on the flap can be seen through a pressure suction peak reduction. The mechanisms responsible for limiting the maximum attainable lift on multielement wing configurations are not well understood. For this reason and in order to find a relation between the physical separation and the unstable modes, the purpose of the current work is to analyze, through a global stability analysis, what occurs before and after the conditions related to a maximum lift condition. Actually, from the point of view of separation, high-lift flow physics is quite complex. Rumsey ${ }^{2}$ described $^{-}$ the possible separation sources, identifying separated flows in many airfoil regions. Each element has its own boundary layer with its transition region where shock/boundary layer interactions and the related boundary layer separations are also possible. The interactions between the different element wakes and the boundary 
layers of the downstream elements is another source of complexity for the high-lift flow field.

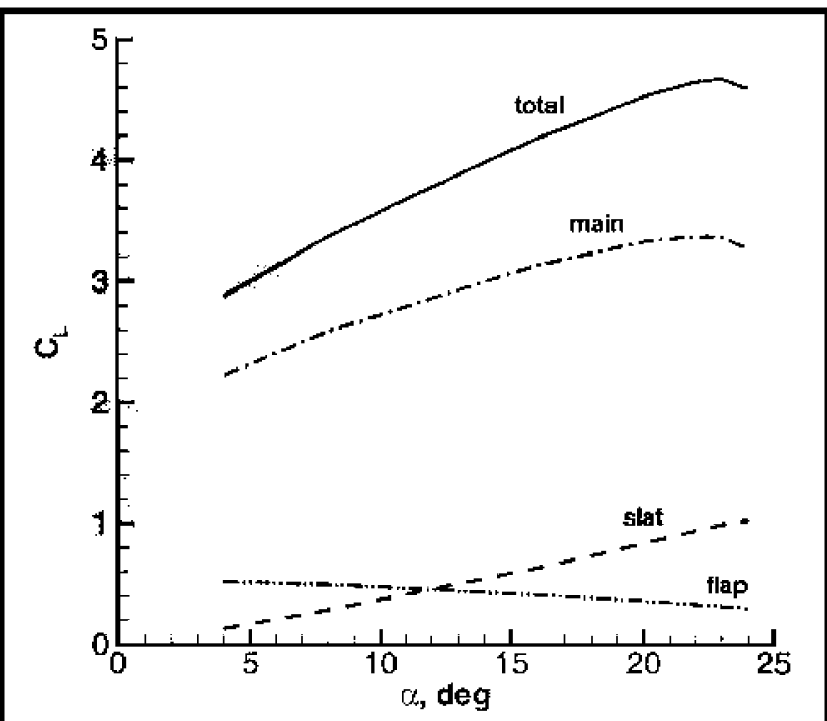

Figure 1. Typical comparison between the lift curves of the different parts and of the complete geometry for a multi-element airfoil.

The use of the stability analysis for this purpose is justified by several successes in detecting shear layer type instabilities. Ehrenstein and Gallaire ${ }^{3,4}$ studied low-frequency oscillations in a separating boundary layer flow, computing nonlinear states of the stationary Navier-Stokes system for a two-dimensional flow over a bump. When increasing the Reynolds number up to values of the order of $10^{2}$, no topological changes in the flow structure were detected, nonetheless, the separation bubble after the bump becomes unstable, with two-dimensional temporal modes represented by localized structures starting at the center of the bubble and extending downstream of the reattachment point.

The stability analysis was also applied to a NACA0012 airfoil in separated conditions by Wales et al. ${ }^{5,6}$ With the continuation method based on the variation of the angle of attack, they found the appearance of an unstable mode just after the $C l_{\max }$ conditions were met. Then, by using a preconditioner based on the Cayley transform to restrict the search area, it was possible to find the rightmost eigenvalues of the system and determine the local stability.

The NACA0012 airfoil was also studied in transonic conditions by Crouch et al. ${ }^{7 / 8}$ They managed to detect, with the Global stability analysis, the onset of the buffet phenomenon, relating the direct unstable mode with the oscillation of the shock wave and of the boundary layer. The study was retreaded by Iorio et al. ${ }^{9}$ who computed the direct and adjoint modes in the same transonic conditions. They were also able to detect the structural sensitivity of the phenomenon giving, in this way, an indication of the modifications needed in the domain region in order to obtain the biggest changes in the flow. The buffet phenomenon was also investigated by Mettot et al. ${ }^{10,11}$ that detected the related direct and adjoint unstable mode and retrieved both the sensitivity gradient to baseflow perturbation and to a steady force on the OAT15A profile 
designed at Onera.

Thus, the idea herein is to extend the concept to more complex and relevant geometries such as highlift geometries, particularly in take-off configuration, and to define with a direct and adjoint analysis the geometrical region where modifications have to be made in order to change the separation conditions.

\section{Mathematical model}

The analyzed problem can be expressed by the Reynolds Averaged Navier-Stokes system of equations in conservative form as:

$$
\frac{\partial}{\partial t} \int_{\Omega} \mathbf{q} d \Omega=-\int_{\partial \Omega} \overline{\overline{\mathbf{F}}} \cdot \mathbf{n} d S
$$

where

$$
\mathbf{q}=\left(\begin{array}{c}
\rho \\
\rho u \\
\rho v \\
\rho w \\
\rho E \\
\rho \widetilde{\nu}
\end{array}\right)
$$

with $\Omega$ being the control volume with boundary $\partial \Omega$, n being the outer normal, and $\overline{\overline{\mathbf{F}}}$ the flux density tensor, whose components can be found $\mathrm{in}^{9} . \rho$ is the fluid density, $\mathbf{v}=(u, v, w)$ are the velocity components and $E=C_{v} T+\left(u^{2}+v^{2}+w^{2}\right) / 2$ is the total specific energy where the product of the specific heat capacity of the gas at constant volume $C_{v}$ and the temperature $T$ represents the thermal specific energy.

The temporal change of the conservative variables $\mathbf{q}$ can be obtained from equation (1) for a control volume fixed in time and space as:

$$
\frac{\partial \mathbf{q}}{\partial t}=-\frac{1}{|\Omega|} \int_{\partial \Omega} \overline{\bar{F}} \cdot \mathbf{n} d S=-\frac{1}{|\Omega|} \mathbf{U}^{F}
$$

with $\mathbf{U}^{F}$ representing the fluxes over the boundaries of the control volume $\Omega$. Discretizing the domain into a finite number of subdomains $\Omega_{i}$ containing $n$ faces each, the temporal variation can be expressed as

$$
\frac{\partial \mathbf{q}_{i}}{\partial t}+\frac{1}{\left|\Omega_{i}\right|} \sum_{j=1}^{n} \mathbf{U}_{j}^{F}=0
$$

Introducing a pseudo-time step $\tau$ in order to solve the steady state case and integrating with the low- 
storage K-step Runge-Kutta scheme, equation (4) becomes:

$$
\Omega_{i} \frac{\partial \mathbf{Q}_{i}}{\partial \tau}+\mathbf{R}_{i}=0 \quad \mathbf{R}_{i}=\sum_{j=1}^{n} \mathbf{U}_{j}^{F}
$$

Where $\mathbf{R}_{i}$ is the residual for the subdomain $i$ and $\mathbf{Q}$ a particular steady solution of the problem. The boundary conditions on the body surface are:

$$
u_{i}=0 \quad \widetilde{\nu}=0 \quad \frac{\partial T}{\partial n}=\frac{\partial \rho}{\partial n}=0
$$

For the external boundaries, a far-field boundary condition is used.

Equation (5) can be written in compact form as:

$$
\mathbf{B} \frac{\partial \mathbf{q}}{\partial t}=\mathbf{R}(\mathbf{q})
$$

where $\mathbf{B}$ is a diagonal matrix with leading dimension $N_{b} N$ that contains the volumes associated to each finite cell, and $N$ is the number of nodes or finite volumes contained in the domain.

Perturbing the basic fluid flow variables with small-amplitude functions $\widetilde{\mathbf{q}}(\mathbf{x}, t)$, these can be written as follows:

$$
\mathbf{q}(\mathbf{x}, t)=\mathbf{Q}(\mathbf{x})+\varepsilon \widetilde{\mathbf{q}}(\mathbf{x}, t)
$$

with $\varepsilon \ll 1$ and $\widetilde{\mathbf{q}}(\mathbf{x}, t)$ can be expressed with explicit harmonic temporal dependence as:

$$
\widetilde{\mathbf{q}}(\mathbf{x}, t)=\widehat{\mathbf{q}}(\mathbf{x}) e^{\omega t}
$$

where $\omega$ is the complex eigenvalue sought and $\widehat{\mathbf{q}}$ describes the complex mode amplitude. In particular, due to the 2D nature of the problem, the Biglobal theory for an infinite spanwise length is applied, thus, the spanwise velocity component and all derivatives in the spanwise direction are neglected. Substituting this formulation in equation ( 7 ) and linearizing, it is possible to obtain the real generalized eigenvalue problem: ${ }^{9}$

$$
\left[\frac{\partial \mathbf{R}}{\partial \mathbf{Q}}\right]_{\mathbf{Q}} \widehat{\mathbf{q}}=\omega \mathbf{B} \widehat{\mathbf{q}}
$$

which can also be expressed as:

$$
\mathbf{A} \widehat{\mathbf{q}}=\omega \mathbf{B} \hat{\mathbf{q}}
$$

where matrix $\mathbf{A}=\left[\frac{\partial \mathbf{R}}{\partial \mathbf{Q}}\right]_{\mathbf{Q}}$ is the Jacobian of the system which is computed once the steady baseflow 
has finally converged. This file is read by the analysis code in order to compute the stability analysis and includes the following boundary conditions:

$$
\widehat{u}=\widehat{v}=\widehat{w}=\widehat{\widetilde{\nu}}=0 \quad \frac{\partial \hat{\rho}}{\partial n}=\frac{\partial \widehat{T}}{\partial n}=0
$$

The number of perturbed variables $N_{v}$ for the turbulent $2 \mathrm{D}$ case is 5 : the density, two velocity components $(\widehat{u}(x, y), \widehat{v}(x, y))$, the energy and eddy-viscosity.

The real diagonal operator $\mathbf{B}$ that represents the volume of each element and the operator $\mathbf{A}$, of dimension $\left(N_{v} N\right)^{2}$, are directly computed by the DLR solver TAU (http://tau.dlr.de/startseite/) and given as an output file.

The complex generalized eigenvalue problem (11) has either real or complex conjugate solutions depending on the imaginary part of the eigenvalue, corresponding to stationary $\left(\omega_{i}=0\right)$ or traveling $\left(\omega_{i} \neq 0\right)$ modes. From a linear stability analysis point of view, the most important eigenvalues are those closest to the axis $\omega_{r}=0$ where the Arnoldi iterative method, already used in several Biglobal linear instability problems, ${ }^{12}$ is used for their determination. In particular, a shift-invert transformation in the Arnoldi algorithm is required to transform the most accurate computational region of the complex plane, originally corresponding to the extreme values part, into the neighborhood of the origin of the complex plane. As described by Iorio et al., ${ }^{9}$ a complete LU factorization was performed for the $\mathbf{A}$ matrix. Hence, the solution of these large linear systems with a sparse real non-symmetric matrix $A$ is performed using a direct LU factorization performed in parallel by the MUMPS library. ${ }^{13}$

In order to determine the structural sensitivity to local feedback, ${ }^{14}$ thus obtaining information about the region where a small perturbation could cause the largest drift in the eigenvalues, ${ }^{15}$ the adjoint modes have to be computed. These can be obtained by solving an eigenvalue system similar to the one previously shown for the direct system in equation (11):

$$
\mathbf{A}_{A d j} \mathbf{q}_{A d j}=\omega_{A d j} \mathbf{B} \mathbf{q}_{A d j}
$$

where $\omega_{A d j}$ and $\mathbf{q}_{A d j}$ denote the adjoint eigenvalues and eigenvectors (i.e. adjoint eigenmodes), respectively.

The discrete adjoint matrix can be expressed as:

$$
\mathbf{A}_{A d j}=\mathbf{B}^{-1} \mathbf{A}^{H} \mathbf{B}
$$

The adjoint eigenvalues $\omega_{A d j}$ are the complex conjugate eigenvalues of the original direct eigenvalue system and the adjoint modes are the left eigenvectors of the original direct matrix A. For this reason, only 
the direct eigenvalue problem needs to be computed since, from its solution, both direct and adjoint modes can be derived.

\section{Results}

The formulation of the linearized forms of the direct and adjoint stability problems expressed in section II was applied to different cases in order to verify the presence of unstable modes for high angles of attack with a low-frequency content with respect to the turbulence. The first case analyzed was the NACA0012 airfoil in subsonic and transonic conditions. The results for the last case of the direct analysis were compared with the analysis of Wales et al. ${ }^{5,6}$ Finally the analysis was also applied to the GARTEUR A310 airfoil, a high-lift test case with slat and flap components in take-off configuration.

For all cases, one unstable mode was detected for an angle of attack greater than the one related to the maximum lift coefficient condition, indicating the gradual tendency of the separated flow to become unstable. Moreover the structural sensitivity was calculated, identifying for each geometry the most sensitive region to change in flow topology.

\section{A. NACA0012 profile in subsonic conditions.}

The first case analyzed was a NACA0012 airfoil in subsonic conditions, with $M=0.15$ and a significant Reynolds number $R e=6 \cdot 10^{6}$ based on the dimensionless chord $c=1$. The computational domain is defined in a plane $\mathrm{XZ}$ with the NACA0012 airfoil located at the center of a circular domain of radius $R=100 \mathrm{c}$. The used mesh is shown in figure 2. The radius of the domain and the number of mesh points were varied until the convergence of the most unstable eigenvalue was reached. Far field boundary conditions were applied to the external boundary and no-slip boundary conditions were used for the airfoil. The RANS simulations were carried out with the steady version of the TAU solver until the maximum residual was less than $10^{-8}$. Several turbulence models were tested and compared with the experimental data available on the NASA webpage. ${ }^{16}$ Figure 3 shows how the $k-\omega$ turbulence model is not able to detect a separated flow, giving wrong results for the $C l_{\max }$ region. The poor performance of the $k-\omega$ models for wall-bounded separated flows caused by adverse pressure gradients was already mentioned by Rumsey. ${ }^{2}$ Both Menter SST and Spalart-Almaras (SA) turbulence models exhibit similar results, giving the maximum $C l_{\text {max }}$ value for a higher angle of attack $\alpha$ when compared to experiments. Since both turbulent models have shown similar results, we choose the baseflow obtained with the SA turbulence model for the global stability analysis. It is important to underline that turbulence models only filter the high frequencies related to the viscous shear 
layer zone, permitting the determination of low-scale frequencies of shear layer type that can be detected by the stability analysis. However the turbulence models provides a mean flow and a Jacobian that still include this physical turbulence as an average.

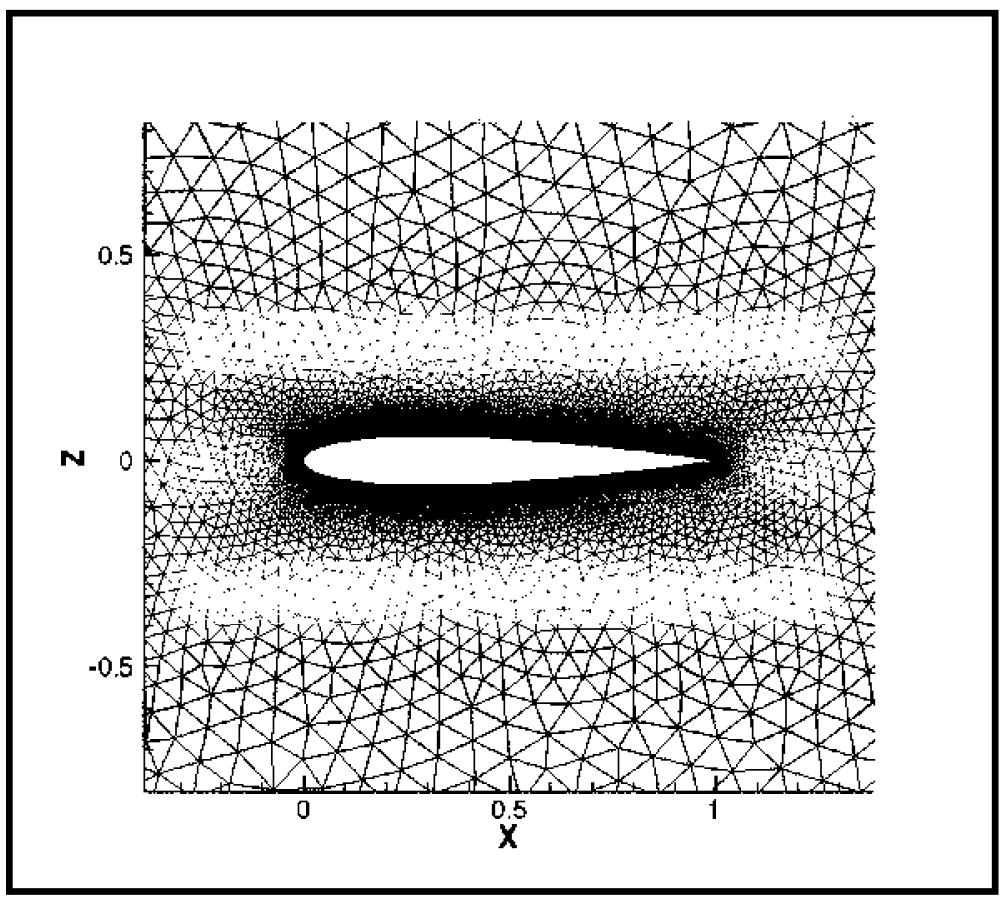

Figure 2. Mesh used for the baseflow and the global analysis of the NACA0012 profile.

The most unstable eigenvalues for different values of the angle of attack $\alpha$ when the computed steady baseflow is perturbed are shown in figure 4. According to the stability criteria and the results plotted in figure 4 , the system becomes unstable at $\alpha=18.9^{\circ}$, greater than the angle of attack $\alpha=18^{\circ}$ associated to the $C l_{\max }$ value indicated by the RANS simulation with the Spalart-Almaras turbulence model. In figure 5, Mach number contours for steady baseflows at $\alpha=18$ (left) and $\alpha=18.9^{\circ}$ (right) are shown and the subsonic regime is confirmed. It is also possible to see how the instability origin is not related to a sudden change in the flow field, but rather to a gradual separation increase.

In order to verify the correctness of these results, a comparison to the ones coming from the unsteady version of the RANS equations (URANS) was done. In fact, unsteady simulations carried out with the TAU solver present convergence to steady values for both the cases $\alpha=18^{\circ}$ (related to the $C l_{\text {max }}$ conditions) and $\alpha=18.8^{\circ}$, while it exhibits an oscillating behavior for $\alpha=18.9^{\circ}$, as represented in figure 6 . The shown results do not seem to be affected when changing the time step size.

The unstable mode is shown in figure 7 in terms of horizontal $\widehat{\rho u}$ and vertical $\widehat{\rho v}$ momentum, energy $\widehat{\rho E}$ and the turbulent viscosity $\widehat{\hat{\rho}}$ of the amplitude of the perturbation. This figure also shows how the instability is related to the separation on the upper part of the airfoil, generating a shear layer instability mode. 


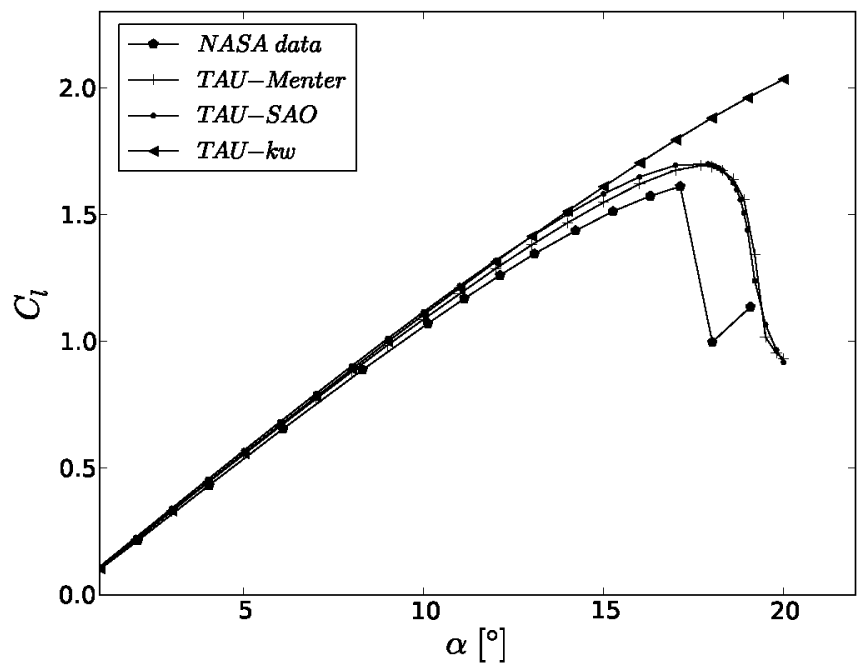

Figure 3. TAU Turbulence model comparison on the $C l$ versus $\alpha$ plot for the NACA0012 at $M=0.15$ and $R e=6 \cdot 10^{6}$.

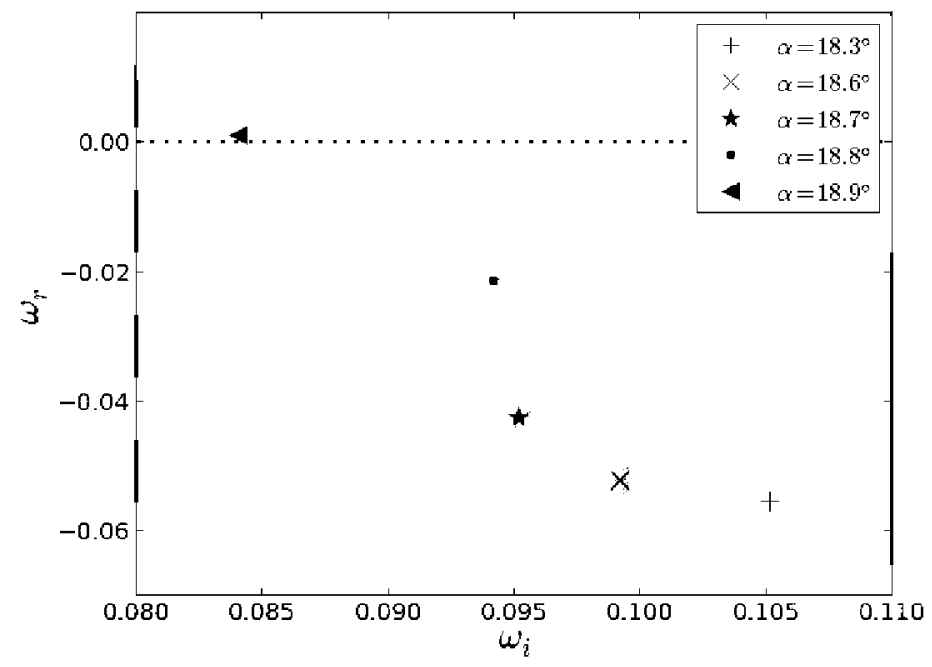

Figure 4. Most unstable eigenvalues obtained from the direct analysis of the NACA0012 in subsonic conditions $\left(M=0.15\right.$ and $\left.R e=6 \cdot 10^{6}\right)$ when increasing the angle of attack $\alpha$. 


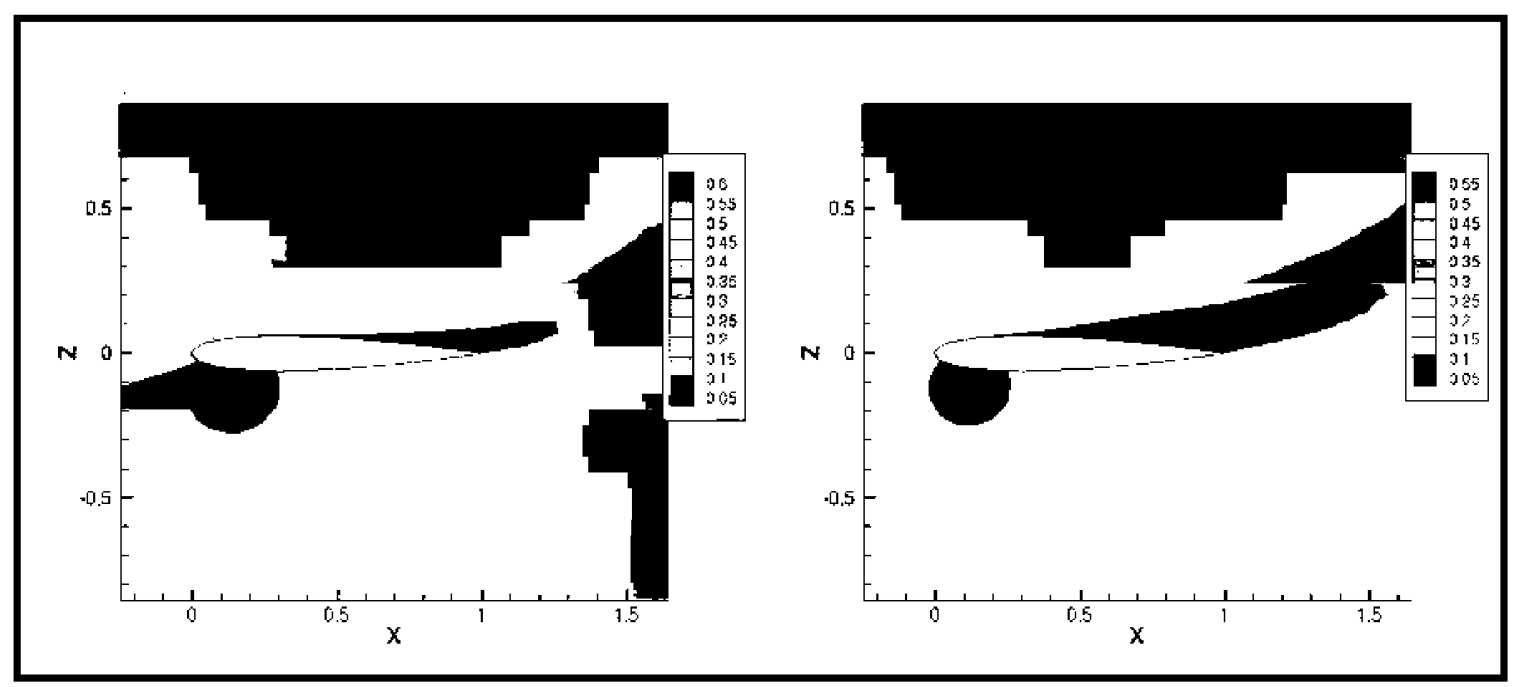

Figure 5. Mach number contour fills for the steady baseflow at $M=0.15$ and $R e=6 \cdot 10^{6}$ for a stable case at $\alpha=18^{\circ}$ (left) and an unstable case at $\alpha=18.9$ (right).
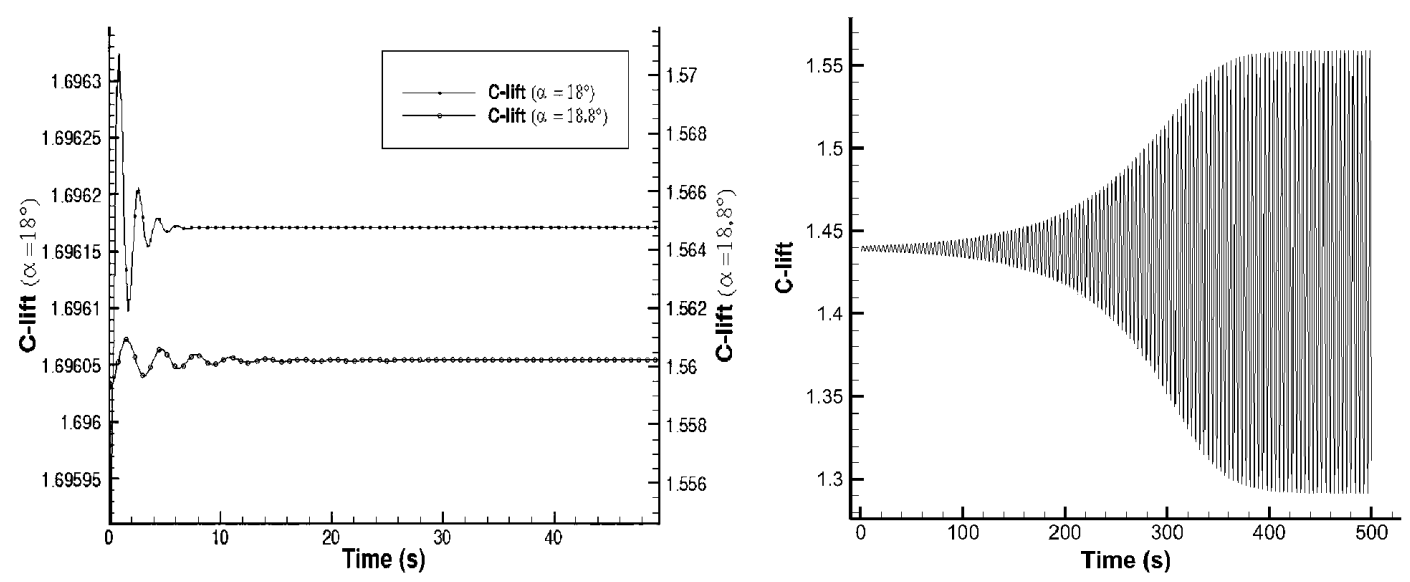

Figure 6. $C l$ variation in time at $M=0.15$ and $R e=6 \cdot 10^{6}$ for $\alpha=18$ and $\alpha=18.8$ (left) and for $\alpha=18.9^{\circ}$ (right) obtained by URANS computations. 

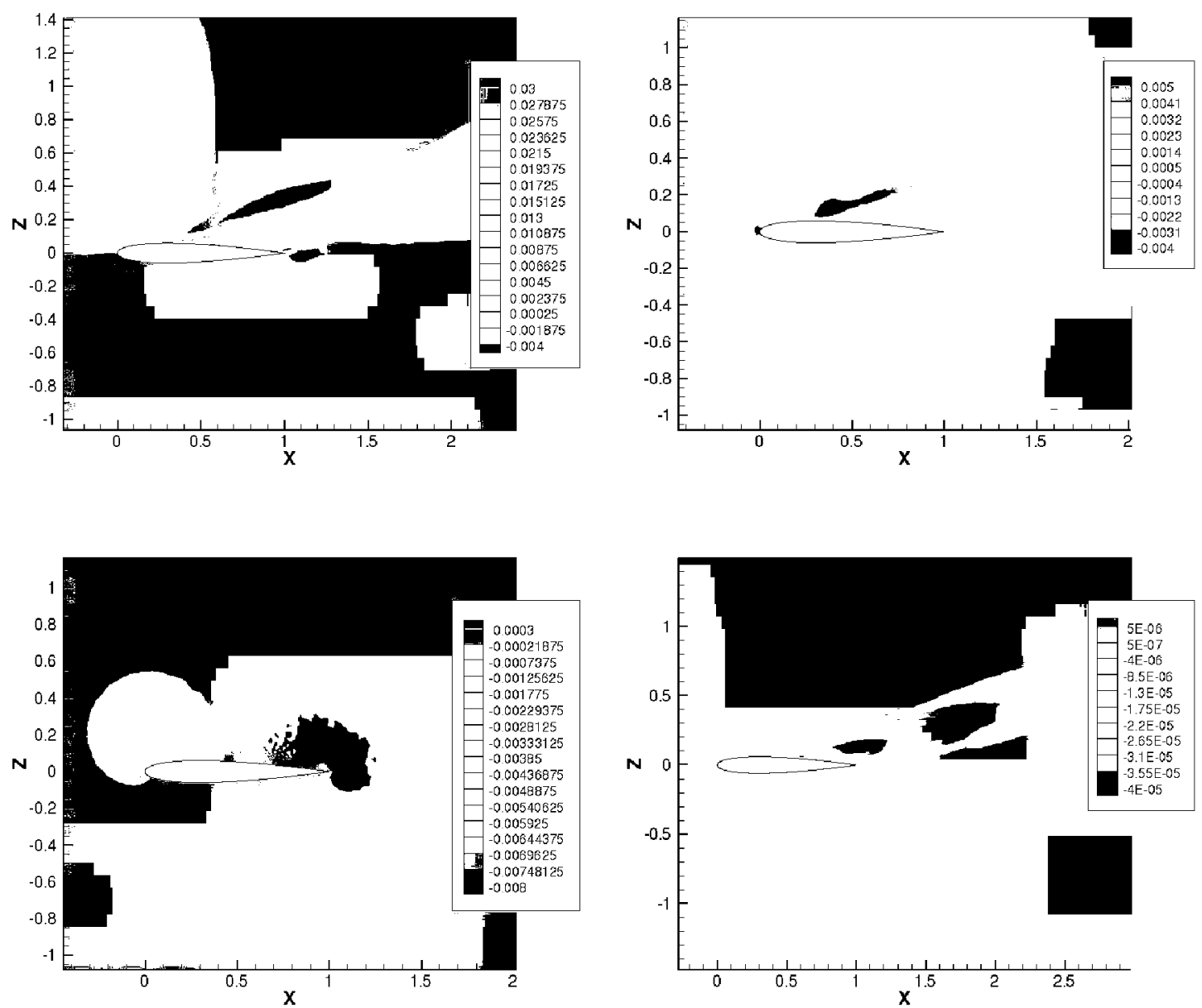

Figure 7. Horizontal $\widehat{\rho} u$ (upper left) and vertical $\widehat{\rho}$ momentum (upper right), energy $\widehat{\rho E}$ (lower left) and turbulent viscosity $\widehat{\rho} \widetilde{\nu}$ (lower right) perturbation amplitudes corresponding to the unstable mode for the NACA0012 at $R e=6 \cdot 10^{6}, M=0.15$ and $\alpha=18.9^{\circ}$.

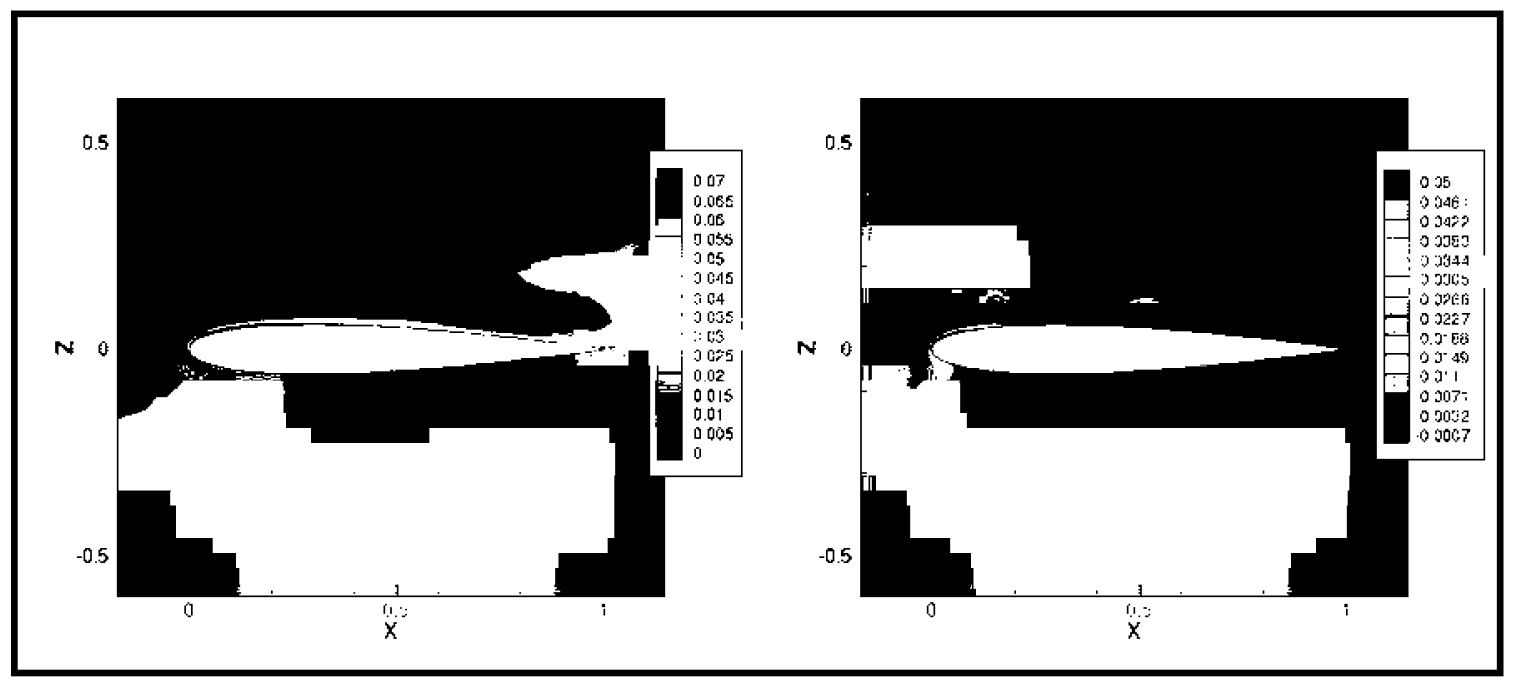

Figure 8. Horizontal $\widehat{\rho u}$ (left) and vertical $\widehat{\rho v}$ momentum (right) components of the most unstable mode of the adjoint formulation for the NACA0012 at $R e=6 \cdot 10^{6}, M=0.15$ and $\alpha=18.9^{\circ}$. 
In figure 8, the amplitude of the perturbation associated with the adjoint mode is plotted. As described in section II, because of the upstreaming nature of the adjoint equations, the perturbation is mainly located before and under the physical separated region on the airfoil. This is an indication of the receptivity region for this type of instability. In fact, in order to obtain the biggest change, an external force should be located in that region.

From the product between the direct and the adjoint mode, the structural sensitivity $S$ could be obtained as $S=\left\|q_{A d j}\right\| \cdot\|\widehat{q}\|$ with $<q_{A d j}, \widehat{q}>=1$ and $\widehat{q}$ and $q_{A d j}$ being the direct and adjoint modes respectively. The structural sensitivity region indicates the region of the domain where an applied force leads to the maximum change in the eigenvalue. As shown in figure 9, changes have to be made on the upper part of the leading edge and on the upper part of the airfoil in front of the start of the flow separation.

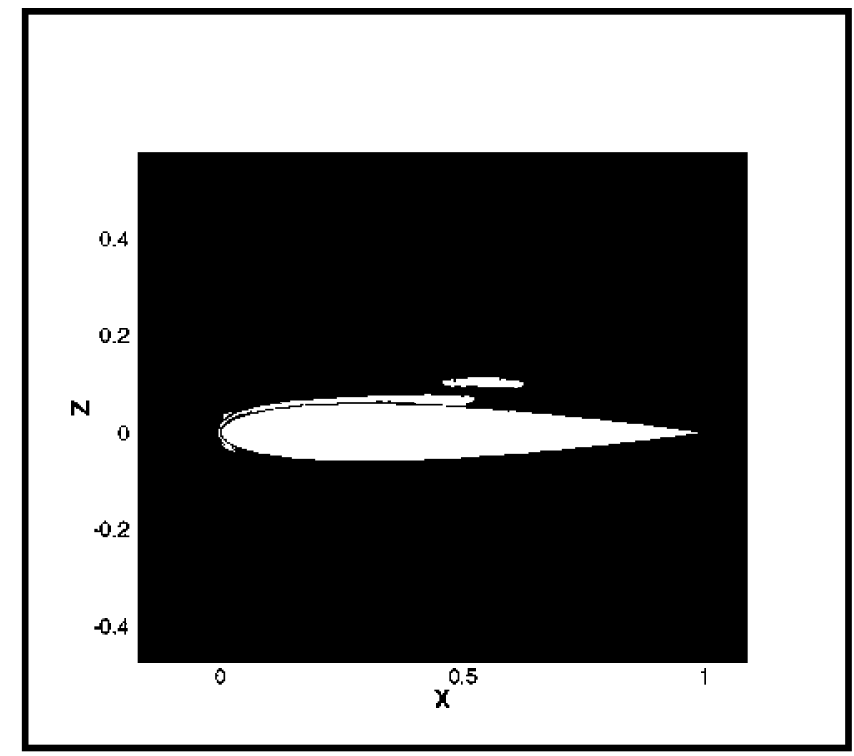

Figure 9. Structural sensitivity map for the NACA0012 at $M=0.15, R e=6 \cdot 10^{6}$ and $\alpha=18.9^{\circ}$.

\section{B. NACA0012 profile in transonic conditions.}

In order to see the changes in the low-frequency unstable mode when a shock wave is present, the NACA0012 airfoil was studied in transonic conditions. Mach number $M=0.5$ and Reynolds number $R e=2.9 \cdot 10^{6}$ were chosen. The same mesh and computational domain used for the subsonic case was selected and the RANS steady simulations were carried out using the previous boundary conditions and the SA turbulence model. In this way, the polar shown in figure 10 was obtained. This steady baseflow solution is also compared to the experiments from AGARD 138 with wall corrections, ${ }^{17}$ and to the polar obtained by Wales et al ${ }^{5} \cdot{ }^{6}$ We can observe in figure 10 that for angles of attack lower than $\alpha=8^{\circ}$, both Wales and TAU computed coefficients are very similar but higher than the experiments. For angles higher than $\alpha=8^{\circ}$, TAU overpredicts the lift 
coefficient values compared to both, Wales computations and experimental results.

The most unstable eigenvalues evaluated with the equation 11 from the baseflow obtained at different values of $\alpha$, are shown in figure 11. In this case, the system becomes unstable for $\alpha=9.5^{\circ}$, identical to the results obtained by Wales. The unstable frequency, in this case, is greater than the one obtained from the subsonic case being now $\omega_{i}=0.46$. The $C l_{\max }$ is located at $\alpha=9^{\circ}$ and, from figure 12 , it is possible to see that the difference in the separated flow between the condition of maximum lift coefficient and the instability onset is quite small, once again indicating the gradual nature of the separation phenomenon that lead to the instability onset.

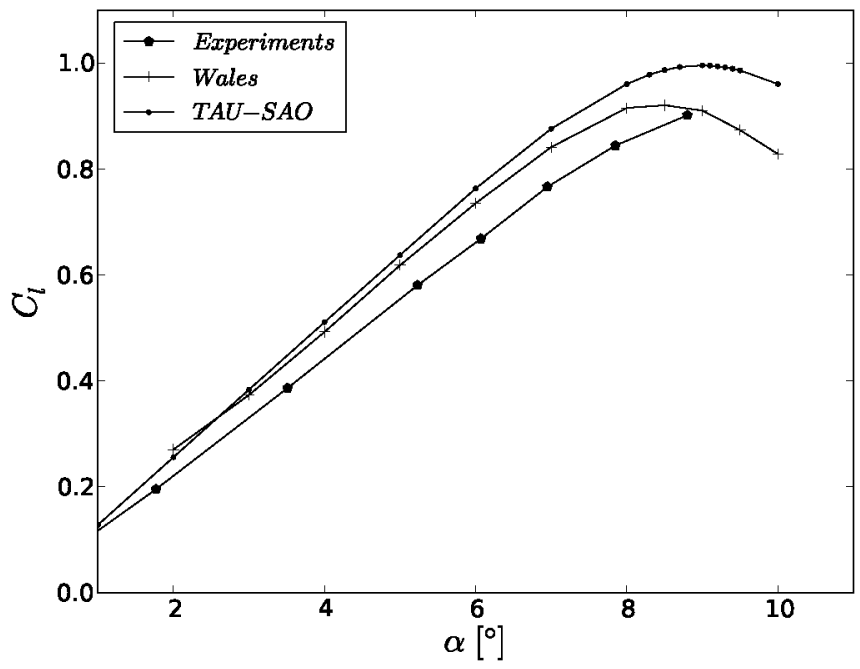

Figure 10. Polar obtained for the NACA0012 at $M=0.5$ and $R e=2.9 \cdot 10^{6}$ using the TAU solver.

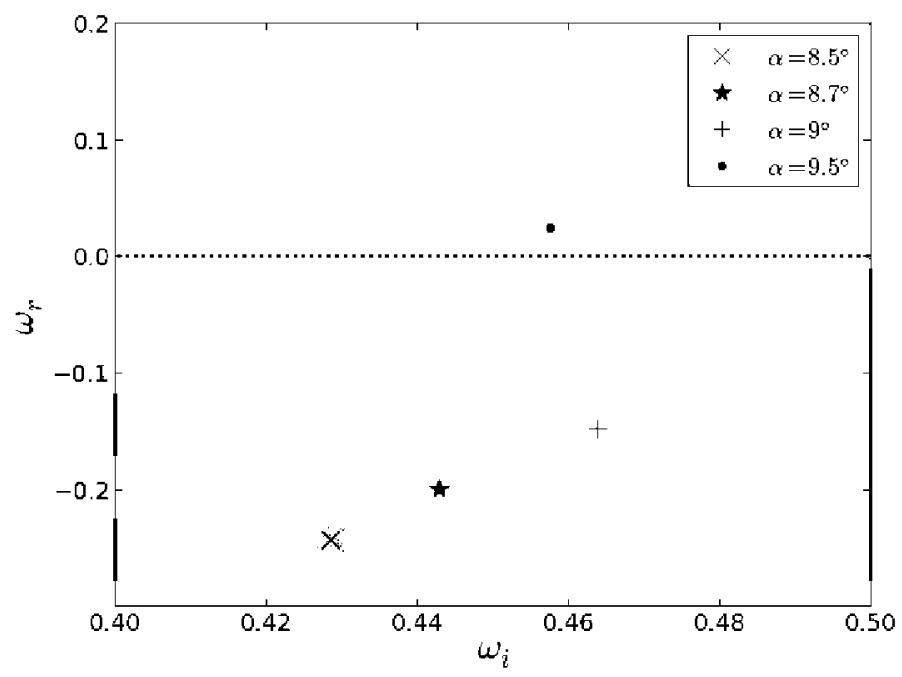

Figure 11. Most unstable eigenvalues obtained from the direct analysis for the NACA0012 in transonic conditions $\left(M=0.5\right.$ and $\left.R e=2.9 \cdot 10^{6}\right)$ when increasing $\alpha$. 

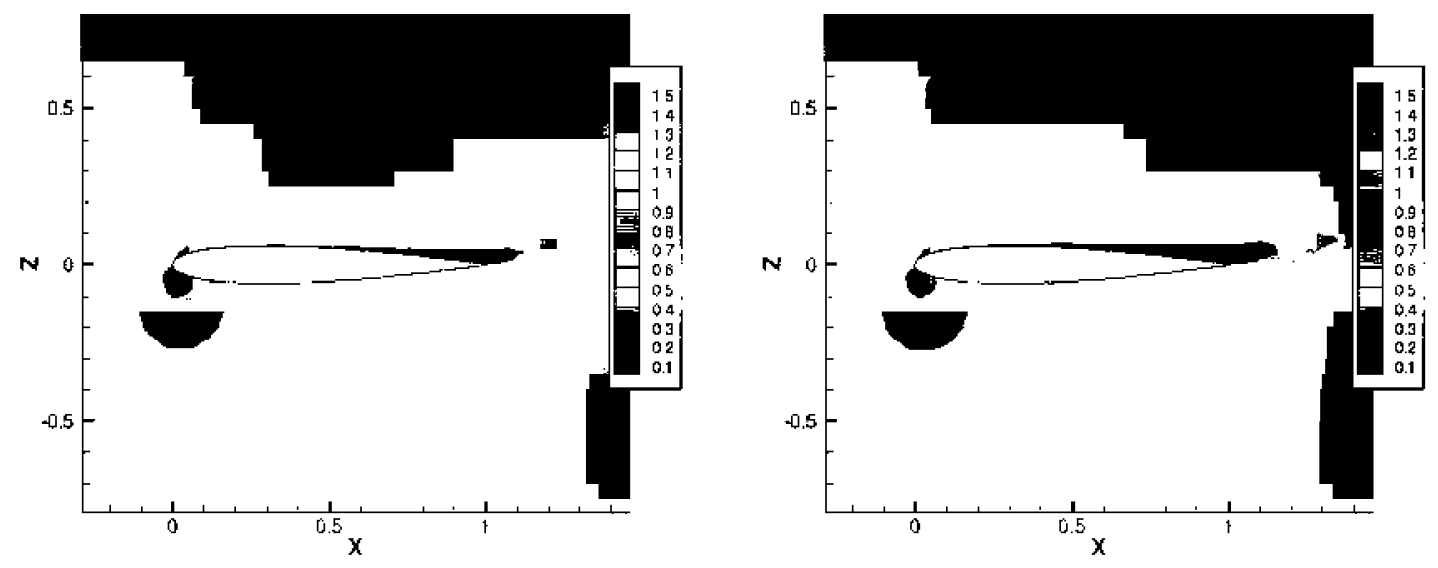

Figure 12. Mach number contour fills for the steady baseflow at $M=0.5$ and $R e=2.9 \cdot 10^{6}$ for $\alpha=9^{\circ}$ (left) and $\alpha=9.5^{\circ}$ (right).

The horizontal $\widehat{\rho u}$ and vertical $\widehat{\rho v}$ momentum, energy $\widehat{\rho E}$ and the turbulent viscosity $\widehat{\rho} \widehat{\bar{\nu}}$ of the perturbation amplitude are shown in figure 13. Due to the shock wave presence and, in this case, the instability starts from the shock wave, provoking a separation along the upper part of the airfoil. A similar result was previously observed in the analysis of the unstable buffet phenomenon of the NACA0012 airfoil, where the amplitude of the perturbation presents the maximum value on the shock wave location and on the upper part of the airfoil..$^{7,9}$

In the same way as described in the previous section, the amplitude of the perturbation associated to the adjoint mode was computed and plotted in figure 14. Due to the upstreaming nature of the adjoint equations, the perturbation is mainly located around the shock wave and extends upstream. This is an indication of the receptivity region; in fact, it is in this region that an external force has to be applied in order to obtain the biggest change in the flowfield.

From the product between the direct and the adjoint mode, the structural sensitivity $S$ could be obtained. $S=\left\|q_{A d j}\right\| \cdot\|\hat{q}\|$ with $\left\langle q_{A d j}, \widehat{q}\right\rangle=1$ and $\widehat{q}$ and $q_{\text {Adj }}$ being the direct and adjoint modes respectively. The structural sensitivity map shown in figure 15 indicates that, in presence of a shock wave, the biggest changes in flow topology are given by changes at the root of the shock, since it is also derived from the sensitivity analysis of the buffet unstable phenomenon. ${ }^{9}$

\section{A310 high-lift test case.}

After the analysis of a single component geometry in different conditions, the idea is to deal with a more complex and realistic geometry from an industrial point of view. The goal is to see if a low frequency instability related to the separation is still present. For this purpose, the A310 airfoil test case in take- 

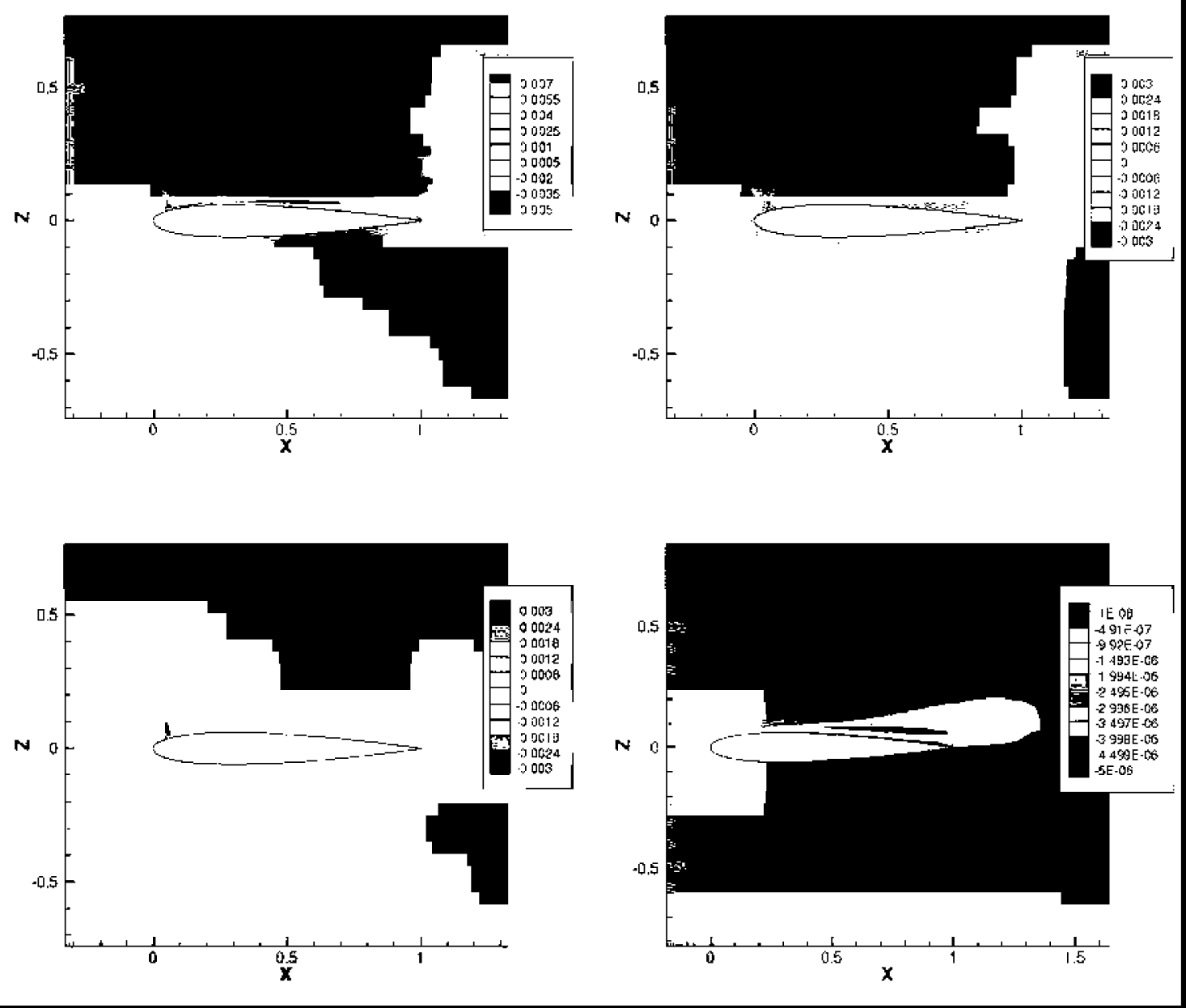

Figure 13. Horizontal $\widehat{\rho} u$ (upper left) and vertical $\widehat{\rho} v$ momentum (upper right), energy $\widehat{\rho E}$ (lower left) and turbulent viscosity $\widehat{\rho} \widetilde{\nu}$ (lower right) perturbation amplitudes corresponding to the unstable mode for the NACA0012 at $R e=2.9 \cdot 10^{6}, M=0.5$ and $\alpha=9.5^{\circ}$.

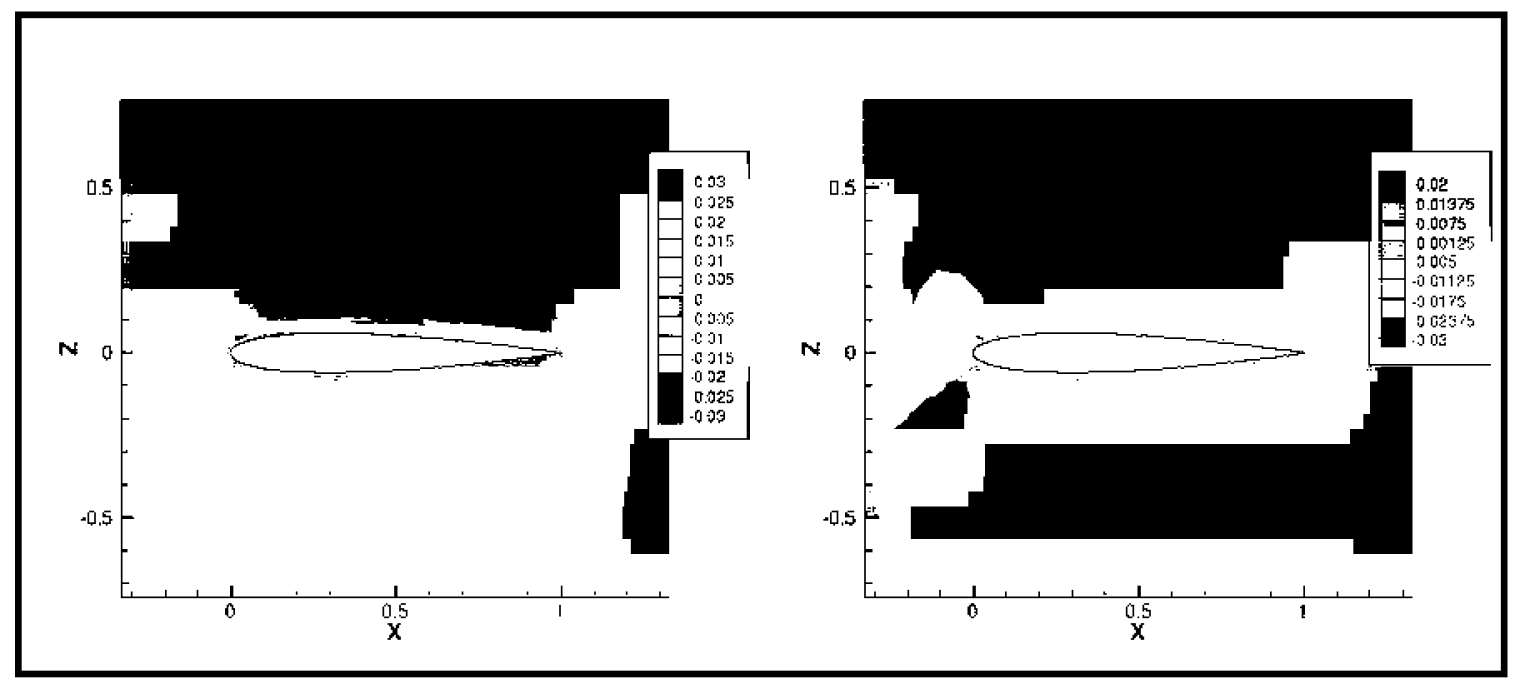

Figure 14. Horizontal $\widehat{\rho u}$ (left) and vertical $\widehat{\rho v}$ momentum (right) components of the most unstable mode for the adjoint formulation for the NACA0012 at $R e=2.9 \cdot 10^{6}, M=0.5$ and $\alpha=9.5^{\circ}$. 


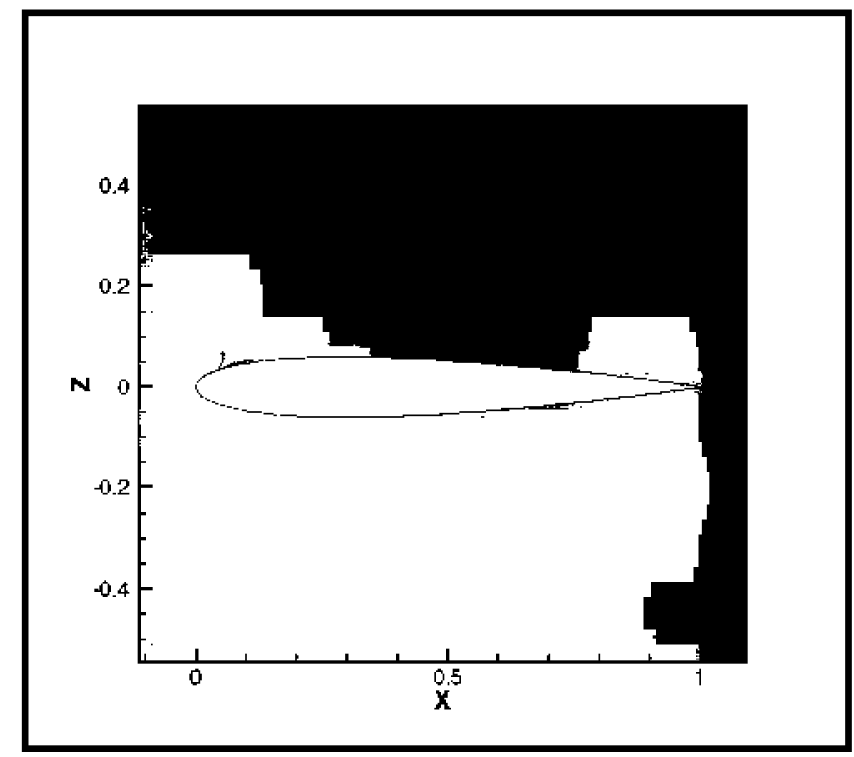

Figure 15. Structural sensitivity map for the NACA0012 at $M=0.15, R e=6 \cdot 10^{6}$ and $\alpha=18.9^{\circ}$.

off configuration was investigated. The polar for $M=0.22$ and $R e=4 \cdot 10^{6}$ was obtained with a RANS simulations and the SA turbulence model using the TAU solver. Even in this case, the computational domain was defined in the XZ plane with the airfoil located in the center of a circular domain of radius $R=100 \mathrm{c}$. The above mentioned polar is compared with the experiments from MBB Transport ${ }^{18}$ in figure 16 , where slightly overpredicted values are found for all the angles of attack, specially on the $\mathrm{Cl}_{\max }$ region.

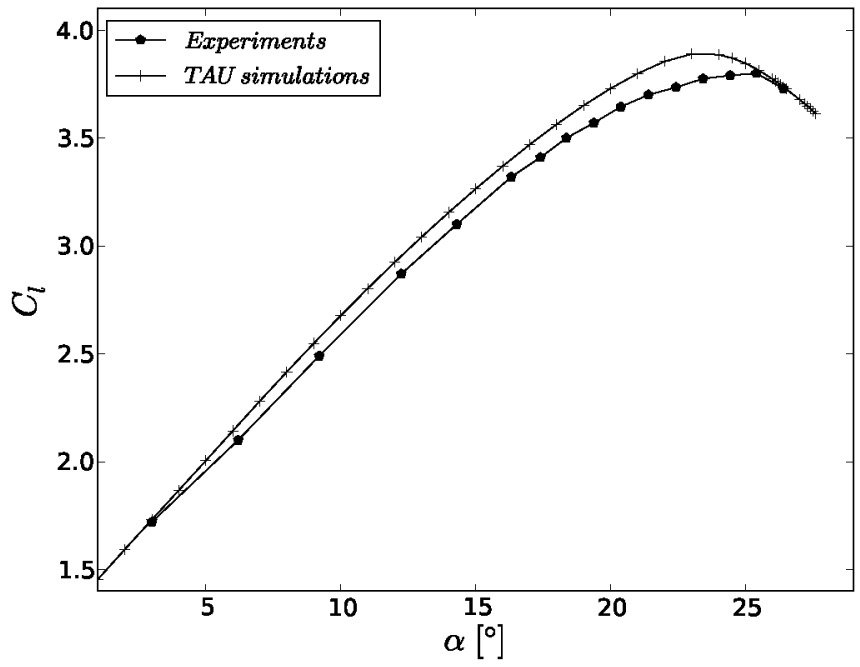

Figure 16. Polar obtained with TAU for the A310 airfoil at $M=0.22$ and $\operatorname{Re}=4 \cdot 10^{6}$.

As before, once the steady baseflow has been computed, the stability analysis was carried out. Three different meshes were used; the meshes $M C 2$ and $M C 4$ were obtained with the mesh generator CENTAUR (http://www.centaursoft.com), while the $A S M$ mesh was obtained with the mesh generator SOLAR ${ }^{19} ;$ mesh 
details are reported in table 1 . In figure 17, it is possible to observe that the critical angle of attack for which the baseflow becomes unstable is close to $\alpha=27.6^{\circ}$. In this situation, a serious mesh convergence process is very expensive in terms of the amount of computer memory consumed during the eigenvalue problem resolution and the long CPU times required for the steady state convergence process near the critical value. In figure 18, it can be seen the instability onset, for two different meshes obtained with SOLAR (ASMref) and CENTAUR (MC4), when the most unstable eigenvalue represented crosses the line $\omega_{r}=0$. When the two extreme meshes are compared, the quantified variation in the critical angle of attack was less than $1 \%$.

\begin{tabular}{|c|c|c|}
\hline Mesh & $n$ & Mesh generator \\
\hline MC2 & 126530 & CENTAUR \\
MC4 & 165392 & CENTAUR \\
ASMref & 255915 & SOLAR \\
\hline
\end{tabular}

Table 1. Different tested meshes obtained with the commercial mesh generators CENTAUR and SOLAR.

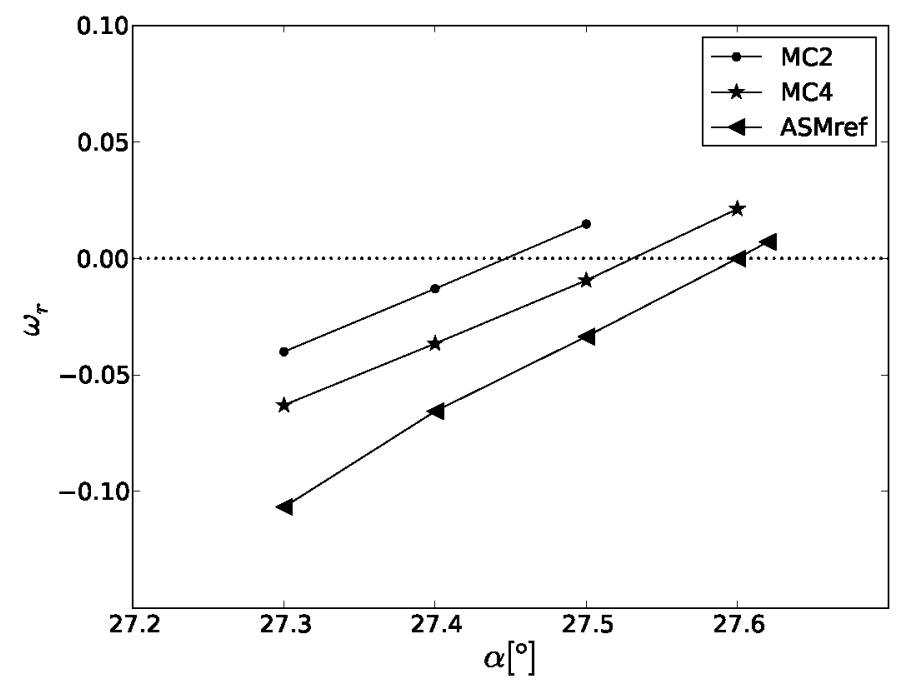

Figure 17. Evolution of the growth/damping rate for the three referenced meshes at different angles of attack for $M=0.22$ and $R e=4 \cdot 10^{6}$. The dotted line separates the stable from the unstable region.

The Mach number contours of the steady flow for the unstable condition are shown in figure 20. These exhibit a supersonic region on the slat surface and a massive separation on the last part of the main airfoil and on the flap.

The instability obtained with the stability analysis is again confirmed by the unsteady computation results. In figure 21, an oscillation period could be observed for the unstable condition at $\alpha=27.6^{\circ}$, when the lift coefficient is plotted versus time.

The unstable mode obtained at $\alpha=27.6$ is shown in figure 22 in terms of horizontal $\widehat{\rho u}$ and vertical $\widehat{\rho v}$ momentum, energy $\widehat{\rho E}$ and the turbulent viscosity $\widehat{\rho} \widetilde{\nu}$ of the perturbation amplitude. From the figure 22 , 

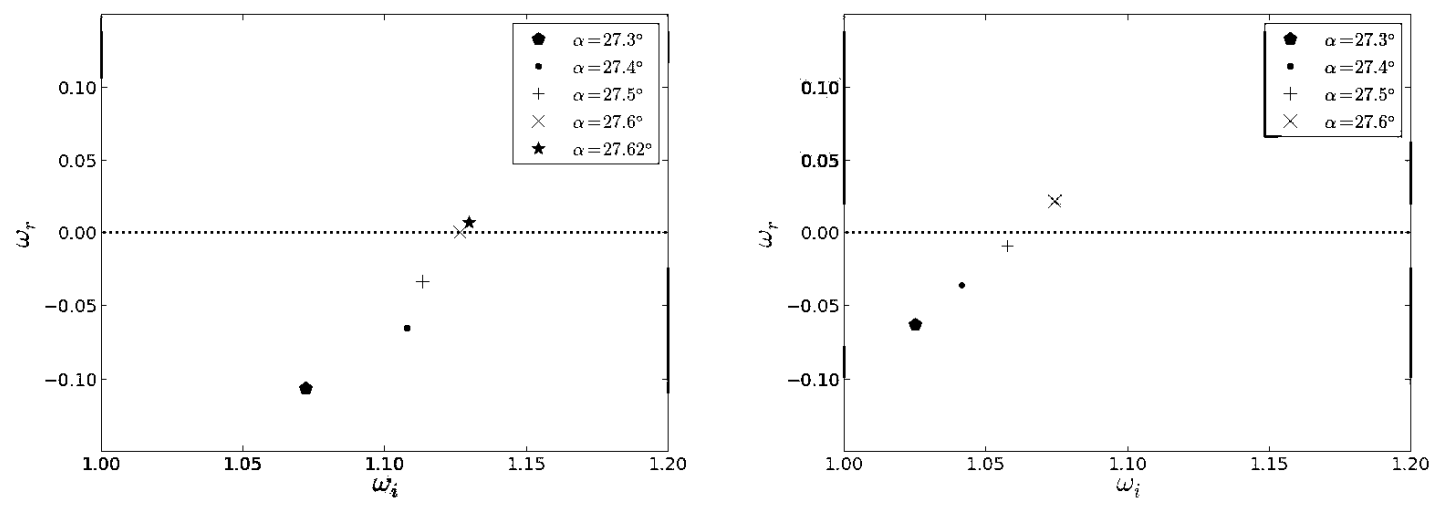

Figure 18. Most unstable eigenvalues obtained from the direct analysis at $M=0.22$ and $R e=4 \cdot 10^{6}$ increasing $\alpha$, for the ASM mesh (left) and the MC4 mesh (right).

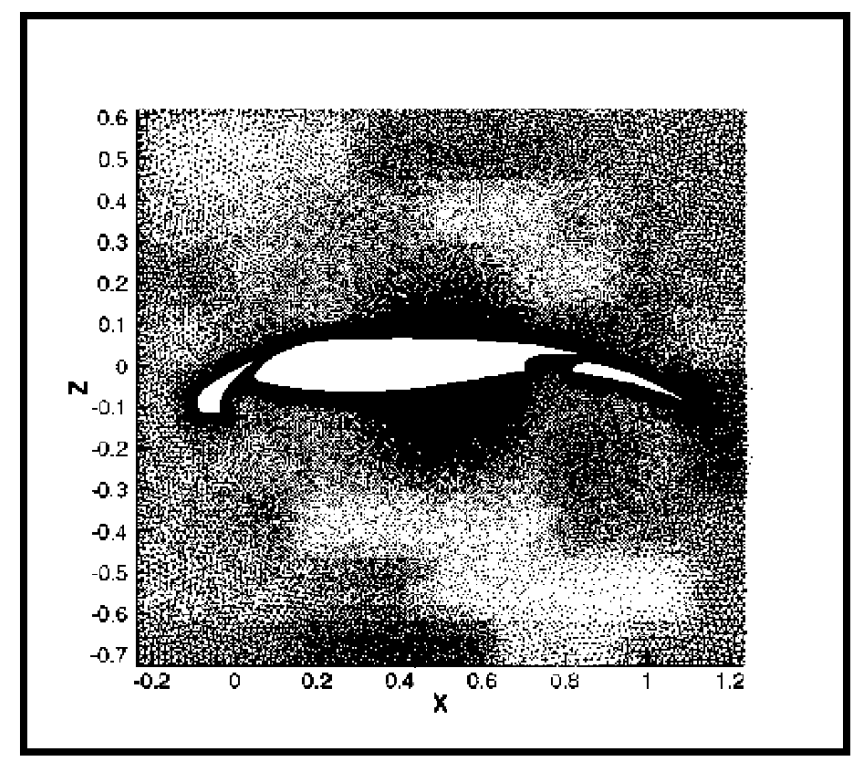

Figure 19. A310 airfoil mesh obtained with CENTAUR. 


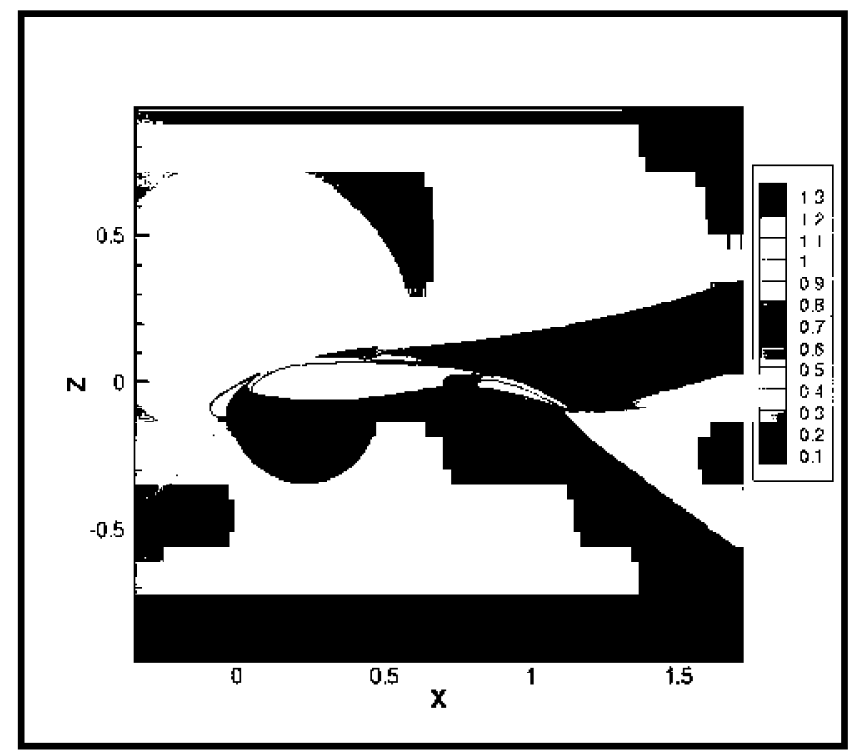

Figure 20. Mach number contours for the steady baseflow at $M=0.22$ and $R e=4 \cdot 10^{6}$ for $\alpha=27.6^{\circ}$.

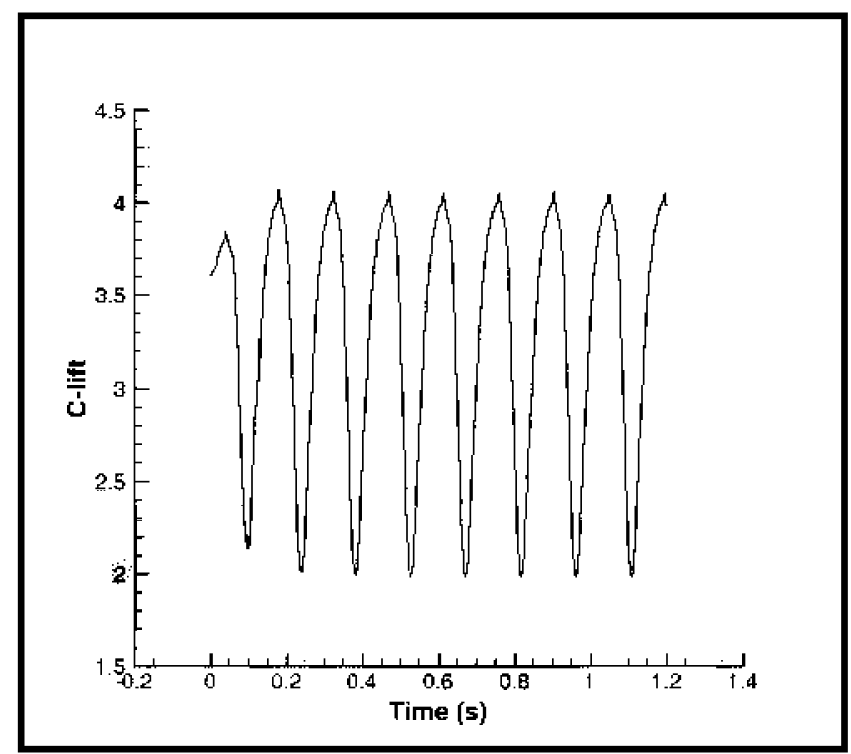

Figure 21. $C l$ variation in time at $M=0.22$ and $R e=4 \cdot 10^{6}$ for $\alpha=27.6$. 
it is possible to see that the instability starts from the trailing edge of the slat, where the beginning of the flow separation can be considered to occur.
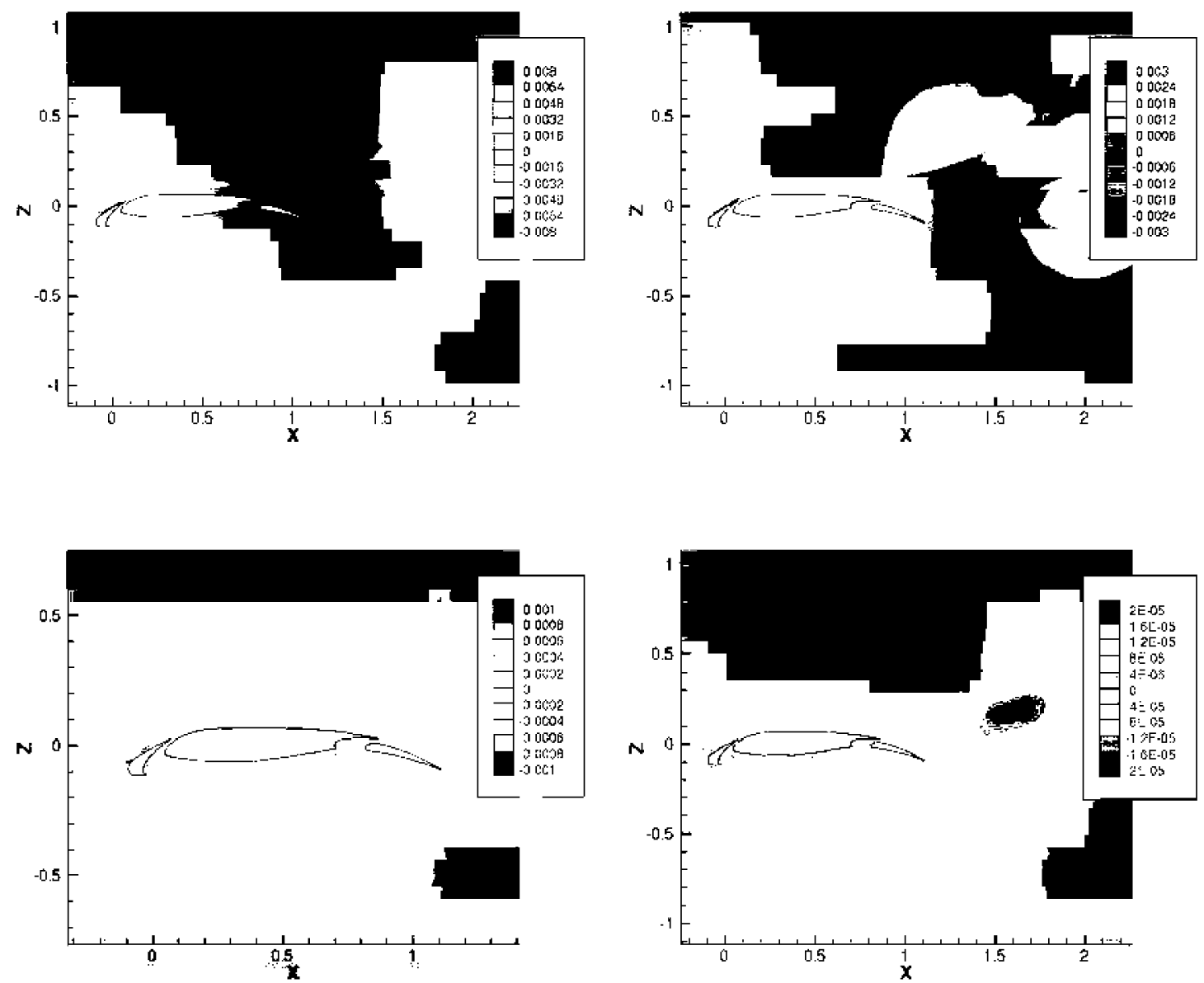

Figure 22. Horizontal $\widehat{\rho u}$ (upper left) and vertical $\widehat{\rho v}$ momentum (upper right), energy $\widehat{\rho E}$ (lower left) and turbulent viscosity $\widehat{\widehat{\rho}}$ (lower right) perturbation amplitudes corresponding to the unstable mode for the $A 310$ airfoil at $M=0.22, \operatorname{Re}=4 \cdot 10^{6}$ and $\alpha=27.6^{\circ}$.

In figure 23, the amplitude of the perturbation associated to the adjoint mode is plotted. In this case, the adjoint perturbation is mainly located on the slat in front of the start of the flow separation, as was expected. Also, from the direct and the adjoint mode for this case, the structural sensitivity was computed. In figure 24 , it can be seen that the region which has to be modified in order to obtain the biggest changes is located on the slat. In particular, in figure 25, a comparison between the sensitivity map and the Mach number contour on the slat is shown. The figure indicates that the sensitivity region starts from the zone in which a supersonic flow is present and extends slightly more along the slat; meaning that, the slat curvature has to be modified in order to avoid the flow oscillation that appears on the separated flow located on the main part of the airfoil. 

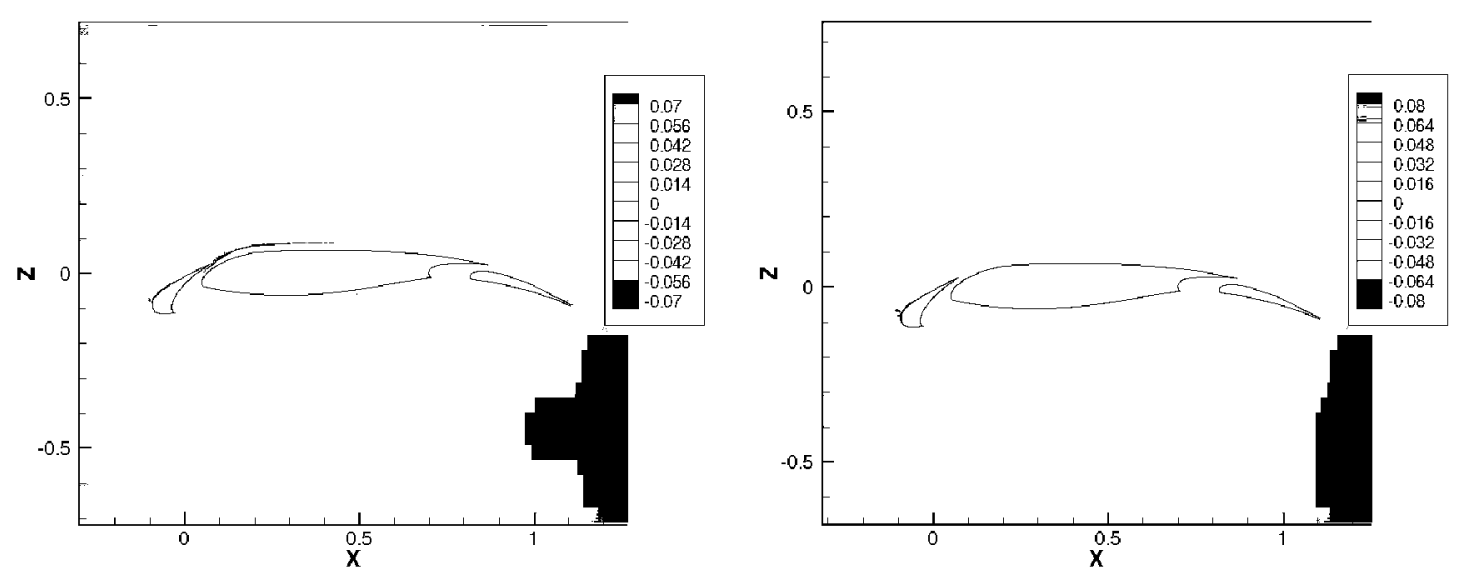

Figure 23. Horizontal $\widehat{\rho u}$ (left) and vertical $\widehat{\rho v}$ momentum (right) components of the most unstable mode for the adjoint formulation.

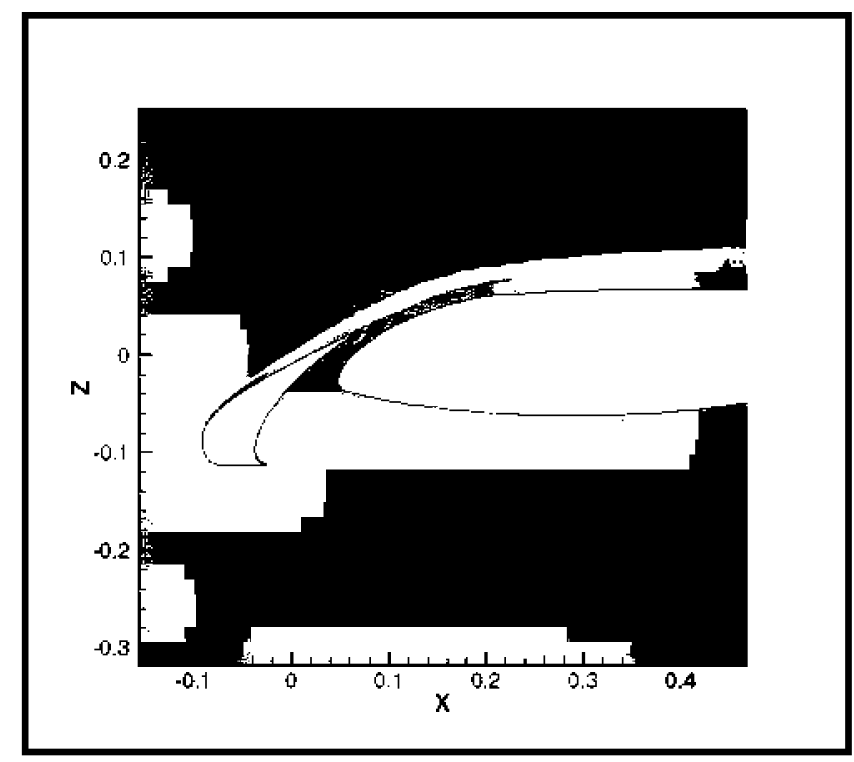

Figure 24. Structural sensitivity map for the $\mathbf{A} 310$ airfoil at $M=0.22$, $R e=4 \cdot 10^{6}$ and $\alpha=27.62^{\circ}$. 

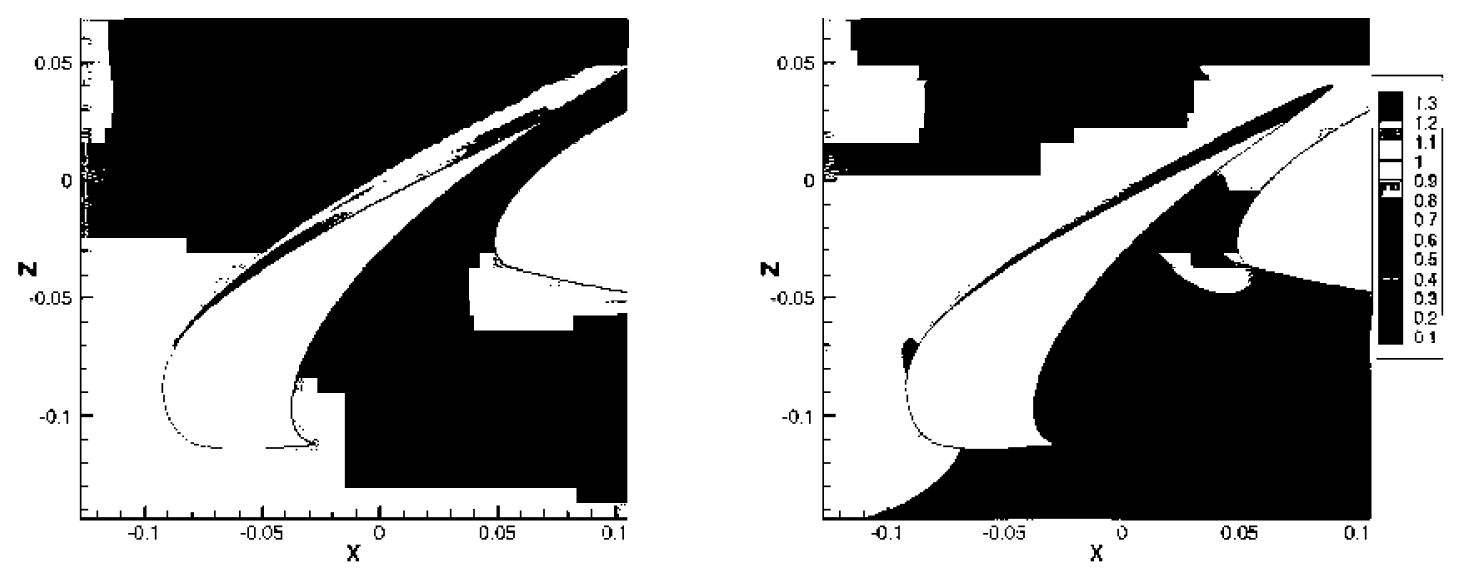

Figure 25. Structural sensitivity map (left) and Mach number contour (right) on the A310 slat in unstable conditions.

A summary table with the corresponding critical angles of attack is shown in table 2 for comparison, where it is possible to appreciate that the values of the critical angles where the instability appears are very different, being the largest the one associated to the A310 geometry.

\begin{tabular}{l|c|c|r}
\hline Geometry & $\operatorname{Re}$ & $\mathrm{M}$ & $\alpha_{\text {crit }}$ \\
\hline NACA0012 & $6 \cdot 10^{6}$ & 0.15 & 18.9 \\
\hline NACA0012 & $2.9 \cdot 10^{6}$ & 0.5 & 9.5 \\
\hline A310 & $4 \cdot 10^{6}$ & 0.22 & 27.6 \\
\hline
\end{tabular}

Table 2. Summary: critical angles and conditions for the different geometries studied.

\section{Conclusions}

The aim of the present work is the study of low-frequency instabilities in turbulent separated flows. The NACA0012 airfoil in subsonic and in transonic conditions, and the take-off configuration of the A310 wing section in flight conditions, were analyzed using global stability analysis. The methodology is comprised of three steps: first, a steady baseflow solution is obtained from the RANS equations, then, the Jacobian matrix of the system is computed, and finally, the generalized eigenvalue problem derived from the perturbed baseflow is solved by an Arnoldi iteration method. The results were compared with the URANS computations showing a good agreement of the instability detection. For all geometries, the direct analysis was carried out by finding the instability onset associated to the separation in turbulent and compressible conditions. This instability appears for all analysed cases at an angle of attack greater than the one related to the $C l_{\max }$ conditions. It was possible too see how, for the NACA geometry, the instability is related to the separation 
starting on the upper part of the airfoil, while, for transonic conditions, this instability depended on the shock wave appearing just after the leading edge. In the high-lift test case, the direct mode starts from the slat, where the separation starts, and extends downstream.

For the first time, both direct and adjoint global stability analyses were carried out for an industrial multi-component A310 airfoil. This complex high-lift configuration was analyzed for turbulent and compressible flows under separated conditions, permitting the detection of the structural sensitivity region that indicates the zone to be modified in order to obtain the biggest change in the flowfield. The results obtained indicate that the region to be modified in order to avoid oscillations related to a separated flow is a small region located on the upper surface of the slat, just after the zone in which the flow accelerates reaching supersonic conditions. It is worth noting that a similar analysis for the NACA0012 at different conditions reports valuable conclusions. While in transonic conditions the region to modify was expected to be the one corresponding to the shock wave, in subsonic conditions it is located on both the upper leading edge and at the point at which the separation starts.

\section{Acknowledgements}

The research leading to these results has received funding from the European project ANADE (Advances in Numerical and Analytical tools for DEtached flow prediction) and from the Spanish Ministry for Science and Innovation under grant TRA2010-16988 "Caracterización Numérica y Experimental de las Cargas Fluido-Dinámicas en el transporte de Gas Licuado". All the authors want to thank Mr. Hugo Gee and Mr. Martin Herring for their valuable assistance during the preparation of this manuscript.

\section{References}

${ }^{1}$ Smith AMO. High-lift aerodynamics. J. Aircraft June 1975; 12(6).

${ }^{2}$ Ramsey CL, Ying SX. Prediction of high-lift: review of present cfd capability. Progress in Aerospace Sciences 2002; (38): $145-180$.

${ }^{3}$ Ehrenstein U, Gallaire F. Global low-frequency oscillations in a separating boundary layer flow. IUTAM Symposium, Unsteady Separated Flows and their Control, Corfu, Greece January 2008; (hal-00213445).

${ }^{4}$ Ehrenstein U, Gallaire F. Two-dimensional global low-frequency oscillations in a separating boundarylayer flow. J. Fluid Mech. 2008; 614:315-327.

${ }^{5}$ Wales C, Gaitonde A, DJones. An initial study of the flow around an airfoil at high Reynolds numbers using continuation. International Journal of Bifurcation and Chaos June 2011; 22(10).

${ }^{6}$ Wales C, Gaitonde AL, Jones DP. Continuation methods applied to the 2D Navier-Stokes equations at high Reynolds numbers. International Journal for Numerical Methods in Fluids January 2012; (70):1258-1289. 
${ }^{7}$ Crouch J, Garbaruk A, Magidov D, Travin A. Origin of transonic buffet on aerofoils. Journal of Fluid Mechanics 2009; 628:357-369.

${ }^{8}$ Crouch J, Garbaruk A, Magidov D. Predicting the onset of flow unsteadiness based on global instability. Journal of - Computational Physics 2007; (224):924-940.

${ }^{9}$ Iorio MC, González L, Ferrer E. Direct and adjoint global stability analysis of turbulent transonic flows over a NACA0012 profile. International Journal for Numerical Methods in Fluids 2014; (10.1002).

${ }^{10}$ Mettot C. Stabilité linéaire, sensibilité et contrôle passif d'écoulements turbulents par différence finies. Thesis presented at the École Polytechnique in Paris 2013; .

${ }^{11}$ Sartor F, Mettot C, Sipp D. Stability, receptivity, and sensitivity analyses of buffeting transonic flow over a profile. $A I A A$ Paper December 2014; (DOI: 10.2514).

${ }^{12}$ González LM, Theofilis V, Gómez-Blanco R. Finite-element numerical methods for viscous incompressible biglobal linear instability analysis on unstructured meshes. AIAA Journal April 2007; 45(4):840-855.

${ }^{13}$ Amestoy PR, Duff IS, L'Excellent JY, Koster J. A fully asynchronous multifrontal solver using distributed dynamic scheduling. SIAM J. Matrix Analysis and Applications 2001; 23(1):15-41.

${ }^{14}$ Giannetti F, Luchini P. Structural sensitivity of the first instability of the cylinder wake. Journal of Fluid Mechanics 6 2007; 581:167-197.

$-{ }^{15}$ Luchini P, Bottaro A. Adjoint equations in stability analysis. Annual Review of Fluid Mechanics 2014; 46(1).

${ }^{16}$ Rumsey C. 2D NACA0012 airfoil validation case. Turbulence Modeling Resource, NASA Langley Research Center; .

${ }^{17}$ Thibert JJ, Grandjacques M, Ohman LH. Experimental data base for computer program assessment. Technical Report 1979; (AGARD AR-138).

${ }^{18}$ Dargel G, Schnieder H. Garteur AD (AG08) final report. Garteur limited distribution (Open from November 2011) November 1989; (MBB Transport - und Verkehrsflugzeuge, Bremen).

${ }^{19}$ Martineau DG, Stokes S, Munday SJ, Jackson AP, Gribben BJ. Anisotropic hybrid mesh generation for industrial RANS applications. AIAA-paper AIAA Aerospace Conference, Reno, USA 2006; (534). 